\title{
The Design of an Miniature Underwater robot for Hazardous Environment
}

\author{
Bin Fang,Wusheng Chou,Xiaoqi Guo, Xin Ma \\ Robotics Institute of Beihang University \\ Beijing, China \\ e-mail: fangbin1120@163.com
}

\begin{abstract}
An miniature underwater robotic system for hazardous environment has been developed. The system consists of an underwater robot, a robot control station and a cameral control station. The underwater robot is installed two cameras for inspection, one is a camera of radiation resistant with two-freedom PTZ in the front of the robot, and the other is a fixed camera in the back of the robot. A miniature manipulator is equipped under the fore-camera to catch the small parts like bolts and nuts in the pools. The movement of the underwater robot is controlled by the master control station and the cameral control station controls the rotation and focus of the fore-camera. Besides, the underwater robot is equipped with the sensors, as MEMS inertial measurement unit, magnetometers, side scan sonar, water-depth gauges, which are integrated to determine the orientation and location of the robot. Meanwhile the navigation information is displayed in the virtual environment, which is modeled upon the real pools of the nuclear power plant. The underwater robotic system is easy to operate and will be applied to the hazardous environment like nuclear environment in future.
\end{abstract}

Keywords-underwater robot; instpection; maintenance; nuclear environment

\section{INTRODUCTION}

Nuclear energy has become an important energy source to worldwide. Meanwhile, the environmental and safety aspects are indispensable to consider. In order to cope with that safety issues related to the nuclear power plants, the uncertain human factors needs to be minimized by automating the inspection and maintenance work done by human workers. The demands of robots in the nuclear industry have been growing to ensure the safety of nuclear facilities, to detect early an unusual condition of it through an inspection, to protect the human workers from an irradiation, and to maintain it efficiently. Therefore, various robots for nuclear environment have been developed ${ }^{[1]}$, and underwater robot is one kind of them.

Then main function of the miniature underwater robot for nuclear environment is inspecting the reactor pressure vessel (RPV), which is the key component of the nuclear power generation system ${ }^{[2]}$. In early nuclear power stations, insufficient consideration was paid to accessibility for inspection personnel, and it was difficult to access the outside surface of the reactor pressure vessel. Periodic access to the outside surface of the RPV by inspectors or maintenance personnel was difficult because such work would require them to pass through a high-radiation area or stay there for some period of time, and because it was difficult to reduce the personnel radiation dose. Nowadays, the solution to this problem is an underwater inspection robot, and there is an urgent need for more thorough inspection of the underwater structures of RPV as an added safety measure, especially after the disaster in Fukushima. Several research projects on underwater robots for nuclear power plants have been reported. KeproVt ${ }^{[3-4]}$ has been developed by the Korea Electric Power Research Institute, and was successfully applied to Yonggwang Nuclear Unit 1 in Korea for the inspection of the lower core plate and baffle plates. In Japan, the underwater inspection robot-AIRIS ${ }^{[5-7]}$ was designed and produced two generations for nuclear facilities, the developed new inspection robot is used in the radioactive waste tanks. Recently, a new version of underwater robot is designed for direct inspection of nuclear power piping systems ${ }^{[8]}$. It is tetherless and compact, but the effectiveness needs to be confirmed in future. [9] also proposes the underwater wireless communication systems in the robotic inspection of nuclear power plants. The technique also should be further verified in practical environment.

According to the requirements of the hazardous environment like nuclear environment, a new underwater robot is developed. The underwater robotic system can not only inspect but also catch the dirty in the pools. In addition, the orientation and location of the robot can be showed to the operator by the virtual environment. The paper is organized as follows. Section 2 presents the functional requirements of the underwater robotic system of the nuclear power plants. Section 3 describes the configuration of the system. Section 4 reports the method of the orientation and location of the robot. Conclusions are drawn in section 5 .

\section{FUNCTIONAL REQUIREMENTS}

Underwater robotic system intended for use within the nuclear environments are subjected to very strict requirements. For the scope of this paper we will focus on three key functional requirements outlined below. They can serve as a valuable starting point for the research and development of prototype direct underwater robotic system.

Compact, smooth shape: For accessing a confined area like the component pools the vehicle must be compact. The dimension of the underwater robot should be miniaturized. Furthermore, the body of the vehicle must be smooth so that the radiant things on the robot would be easy to washed by the clean heavy-pressure water after fulfill the tasks.

Radiation resistant: Though the water provides sufficient shielding against radiation, high level radiation in the reactor vessels. The radiation hardening strategy should be taken. Moreover, the video for inspection should be clear. 
Safe: Safe is the most important. When the underwater robot is applied in the nuclear environment, the safe actions should be considered. The vehicle must maneuver in low speed, and should be controlled accurately by the operator. Meanwhile the location of the vehicle should be showed to help the operator control the robot. Furthermore, the worst situation needs to be considered. In case the robotic system happens to be failure, the vehicle should be recycled by the operator.

This paper proposes the design of our underwater robotic system that addresses all three of these important requirements. The details of the system will be described in the next section.

\section{SYSTEM CONFIGURATION}

The underwater robotic system for hazardous envrioment is composed of an underwater robot, a master control station and a cameral control station.

\section{A. Underwater Robot}

The design prototype of the underwater robot is shown in Fig.1. The main frame of the underwater robot is made of stainless steel and anodized aluminum, so that the robot can resistant to acid in the pools and bear the enough load. On the top of robot, yellow foamed buoyant block is arranged to provide the float of the robot. The shape is designed by the calculation and simulation in ADAMS, in order to keep the vehicle balance in the water. In the front of the block, side-scan sonar is set up. In the middle of robot, the control cabinet is mounted. The cabinet is made of stainless steel and the inner wall put the lead sheet to protect the circuit boards and electronics. In the back of the cabinet, a common camera and five small lights are equipped. It is a CCD camera, so it cannot work long time in the high radiation environment. A high radiation resistant camera is arranged in the front of the robot. The camera that is supplied by IST-REES guarantees performance up to $10^{5} \mathrm{rad} / \mathrm{h}$ radiation dose rate and $10^{8} \mathrm{rad}$ radiation tolerance. Meanwhile, two lights are equipped besides the camera and the two-freedom PTZ provides the more scope of inspection. Then the underwater robot can inspect long hours in the pools of the nuclear power plant. To equal the task of maintenance, a miniature manipulator is mounted under the fore cameral, and the different grippers can be changed in the end of manipulator in according to the different tasks.

There are four thrusters in the underwater robot. It has two vertical thrusters and two horizontal thrusters. The thruster pairs are allocated to be parallel in the vertical or horizontal plane. The allocation of thrusters permits the robot to have four degrees of freedom. In accordance with the rotating direction, the vertical thrusters make the robot move up and down or roll, and the horizontal thrusters make the robot move back and forth or rotationally. Thus, the robot dynamics can be decomposed into up and down or roll behavior, and back and forth or heading behavior. Accordingly, the vertical thrusters VR and VL, and horizontal thrusters $\mathrm{HR}$ and HL can be controlled separately.

The underwater robot's power transmission and communication are in charge of the zero buoyancy cable, which is floated in the water. Besides, the fore cameral has own cable to transmit the video.

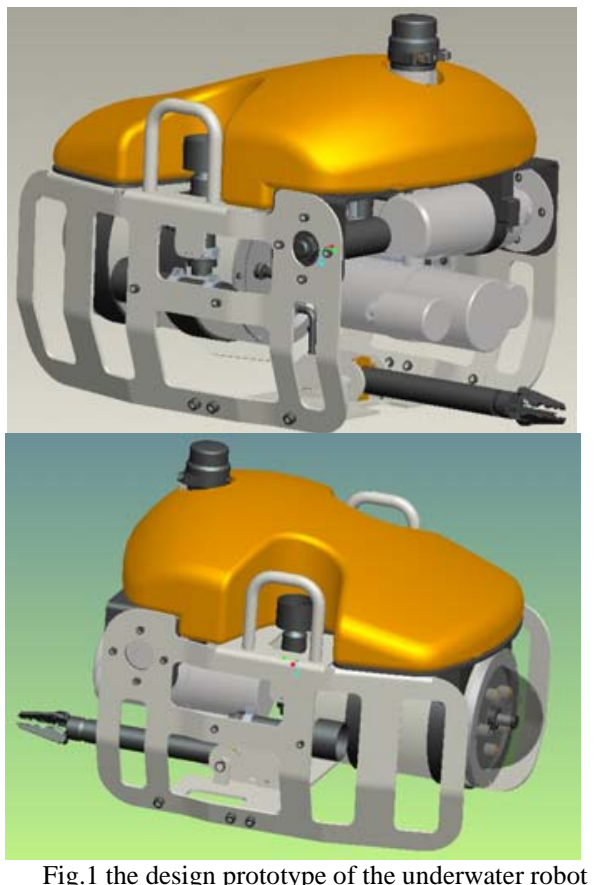

The specification of the underwater robot is listed in Table.1. The design of the underwater robot meets the requirements of the nuclear power plants.

Table 1 specification of underwater robot

\begin{tabular}{|c|c|}
\hline \multicolumn{2}{|c|}{ Specification } \\
\hline Dimensions & $\begin{array}{c}500 \mathrm{~mm} \times 400 \mathrm{~mm} \times 395 \mathrm{~mm} \\
(\mathrm{~L} \times \mathrm{W} \times \mathrm{H})\end{array}$ \\
\hline Material & $\begin{array}{c}\text { stainless steel Anodized } \\
\text { aluminum foamed buoyant material }\end{array}$ \\
\hline Weight (air) & $\begin{array}{c}26.9 \mathrm{~kg} \\
\text { Thruster installation }\end{array}$ \\
\hline Camera & $\begin{array}{c}\text { Two vertical and two } \\
\text { horizontal thrusters }\end{array}$ \\
\hline Light & $\begin{array}{c}\text { Common camera in the back } \\
\text { R981 camera in the front }\end{array}$ \\
\hline Sensors & $\begin{array}{c}\text { Two lights in the front, five } \\
\text { small lights in the back }\end{array}$ \\
\hline Miniature Manipulator & $\begin{array}{c}\text { Depth sensor, scan sonar, } \\
\text { MIMU, magnetometers }\end{array}$ \\
\hline
\end{tabular}

\section{B. Robot control station}

The robot control station is composed of an industrial PC and a robot master control cabinet. There are two ways to control the underwater robot. The one way is realized by the user interface. A series of utility programs such as robot movement control program, robot position and orientation 
measuring program, video record program are installed in the PC. The video images of the inspection cameras are sent to the frame grabber in the PC and get an overlay text image which indicates job identification, date, location of image, and so on. The other way is realized by the robot master control cabinet. The movements of the underwater robot are controlled by two rocker bars. Meanwhile, automatic mode of depth control can be selected in the control station. Upon selecting the automatic operation mode of the depth control, the robot can keep the setting depth in the water.

The robot control station generates commands and sends them to the robot through the zero buoyancy cable. For the inspection and maintenance of the pools of the nuclear power plants, the control station is placed in the safe control room to get rid of the radiation for the operators.

\section{Camera control station}

The fore camera is solely controlled by the camera station. The focal length of the lens and the diameter of the aperture are adjusted by the camera control station. Besides, the PTZ and two servo lights are also controlled by the station. The servo lights can better supply brightness for inspection, and the two freedom of the PTZ can expand the scope of the vision.

\section{Prepare Your Paper Before Styling}

In order to provide the working state of the underwater robot to the operator, the navigation sensors included MEMS accelerometers, MEMS gyros, magnetometers, water-depth gauges, side scan sonar are equipped to determine the positions and orientations of the vehicle. The accelerometers, gyros and magnetometers are set in the control cabinet, the acceleration and angular can be measured. When the vehicle is static, the roll, pitch and heading can be computed by the three sensors. The depth of the robot is measured by the water-depth gauges, whose measurements contain noises. The preprocessing is implemented to improve the accuracy. The side scan sonar is measuring the distance between the vehicle and the wall. The data of the sonar are mapping, and then the matching algorithm is implemented to attain the positions of the plane. In the end, the information of the sensors is integrated in the Kalman filter, the optimal results of the positions and orientations are determined. The algorithm flow is shown in the Fig.2.

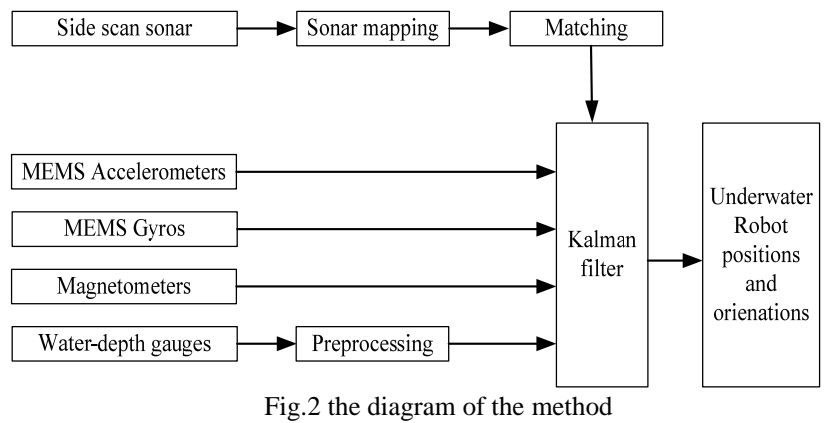

To verify the method validness, the experiment of the underwater robot is implemented in the pool, as shown in Fig.3.
The position and orientation of the robot are measured with the measuring unit with resolution of $\pm 5 \mathrm{~cm}$ and \pm 1 .

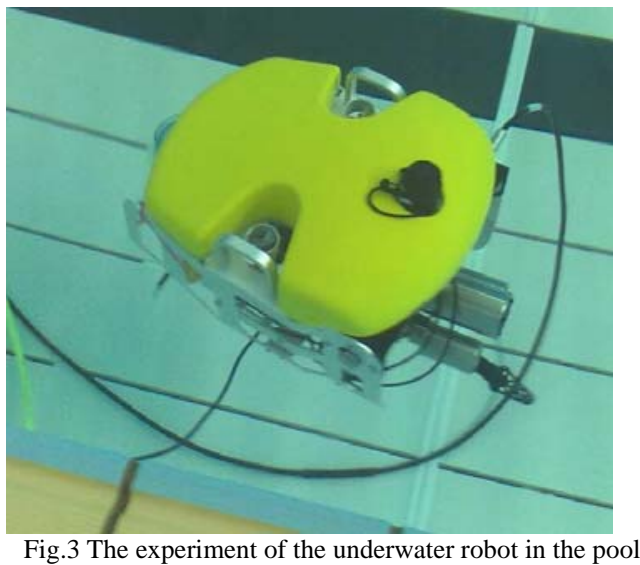

Moreover, the virtual operated environment can be modeled. Then the virtual model of the underwater robot is real-time shown in the environment by the estimated positions and orientations. Consequently, the operator would acquire the situation of the underwater robot and would be easy to operate to inspect and maintain in the hazardous environment.

\section{CONCLUSION}

A miniature underwater robotic system has been developed for inspection and maintenance in the hazardous environment like nuclear environment. The requirements of the nuclear environment have been fully considered, and the robot has been designed to be compact, streamline and high radiation resistant. The system is easy to operate and can be set up for operation in a short time. The underwater robotic system has been verified the effectiveness in the common environment and will be applied to the nuclear environment in future.

\section{ACKNOWLEDGMENT}

The authors are grateful for the support of China Program on the project of International Thermonuclear Experimental Reactor (ITER) under grants number 2012GB102006.

\section{REFERENCES}

[1] M. Odakura,Y. Kometani,M. Koike,M. Tooma, Y. Nagashima. "Advanced Inspection Technologies for Nuclear Power Plants", Hitachi Review, vol. 58, pp.82-87,2009

[2] S. Kim, S. Ho Jung, S. Uk Lee,etc. “Application of Robotics for the Nuclear Power Plants in Korea”. 2010 1st International Conference on Applied Robotics for the Power Industry Delta Centre-Ville Montréal, Canada, October 5-7, 2010:1-5

[3] B.H. Cho, S.H. Byun, C.H. Shin, J.B. Yang etl. "KeproVt: underwater robotic system for visual inspection of nuclear reactor internals”. Nuclear engineering and design, vol.231, pp.327-335,2004

[4] J.Y. Park, B.H. Cho, J.K. Lee.“Trajectory-tracking control of underwater inspection robot for nuclear reactor internals using time delay control”. Nuclear engineering and design,vol.239, pp.25432550, 2009

[5] K. Koji. "Underwater inspection robot-AIRIS 21". Nuclear Engineering and Design,vol 188, pp.367-371,1999 
[6] Y. Miwa, S. Satoh, N. Hirose. "Remote controlled Inspection Robot for Nuclear facilities in Underwater Environment”, ICONE10, Arlington VA USA, 2002

[7] H. Okihana, K. Iwata, Y. Miwa. "Remote controlled inspection robot for nuclear facilities in underwater environment”, Proceedings of the 17th international conference on nuclear engineering, July 12-16, 2009, Brussels, Belgium: 1-5.

[8] A. Mazumdar, M. Lozano, A. Fittery, H. H. Asada. A Compact, Maneuverable, Underwater Robot for Direct Inspection of Nuclear
Power Piping Systems. 2012 IEEE International Conference on Robotics and Automation River Centre, Saint Paul, Minnesota, USA May 14-18, 2012: 2818-2823

[9] Ian C. Rust, H. Harry Asada. A Dual-Use Visible Light Approach to Integrated Communication and Localization of Underwater Robots with Application to Non-Destructive Nuclear Reactor Inspection. 2012 IEEE International Conference on Robotics and Automation River Centre, Saint Paul, Minnesota, USA May 14-18, 2012: 24452450 